\title{
Site-Specific Recombination in the Cyanobacterium Anabaena sp. Strain PCC 7120 Catalyzed by the Integrase of Coliphage HK022
}

\author{
Olga Melnikov, ${ }^{1 *}$ Arieh Zaritsky, ${ }^{1}$ Aliza Zarka, ${ }^{2}$ Sammy Boussiba, ${ }^{2}$ \\ Natalia Malchin, ${ }^{3}$ Ezra Yagil, ${ }^{3}$ and Mikhail Kolot ${ }^{3}$ \\ Department of Life Sciences, Ben-Gurion University of the Negev, P.O. Box 653, Be'er-Sheva 84105, Israel ${ }^{1}$; \\ Microalgal Biotechnology Laboratory, Blaustein Institute for Desert Research, Ben-Gurion University at Sede-Boker, \\ Sede-Boker 84990, Israel ; and Department of Biochemistry, Tel-Aviv University, Tel-Aviv 69978, Israel ${ }^{3}$
}

Received 17 March 2009/Accepted 27 April 2009

\begin{abstract}
The integrase (Int) of the $\lambda$-like coliphage HK022 catalyzes the site-specific integration and excision of the phage DNA into and from the chromosome of its host, Escherichia coli. Int recognizes two different pairs of recombining sites $a t P \times a t t B$ and $a t t L \times a t t R$ for integration and excision, respectively. This system was adapted to the cyanobacterium Anabaena sp. strain PCC 7120 as a potential tool for site-specific gene manipulations in the cyanobacterium. Two plasmids were consecutively cointroduced by conjugation into Anabaena cells, one plasmid that expresses HK022 Int recombinase and the other plasmid that carries the excision substrate $P_{\text {gln }}$-attL-T1/T2-attR-lacZ, where T1/T2 are the strong transcription terminators of $r r n B$, to prevent expression of the $l a c Z$ reporter under the constitutive promoter $P_{g \ln A}$. The Int-catalyzed site-specific recombination reaction was monitored by the expression of lac $Z$ emanating as a result of T1/T2 excision. Int catalyzed the site-specific excision reaction in Anabaena cells when its substrate was located either on the plasmid or on the chromosome with no need to supply an accessory protein, such as integration host factor and excisionase (Xis), which are indispensable for this reaction in its host, $E$. coli.
\end{abstract}

The site-specific recombination mechanism of the temperate coliphage HK022 is similar, if not identical, to that of phage $\lambda$. To lysogenize its Escherichia coli host, circular phage DNA recombines via a site-specific recombination reaction between the nonidentical attP and $a t t B$ sites of the phage and host genomes, respectively. This reaction results in the integrated prophage that is flanked by the recombinant $a t t L$ and $a t t R$ sites, which are the sites for phage excision. The phage-encoded site-specific integrase (Int), which belongs to the Tyr recombinase family, catalyzes phage integration and excision. Both reactions require the presence of the host-encoded DNA-bending heterodimeric accessory protein integration host factor (IHF); excision requires in addition the phage-encoded excisionase (Xis), whose activity can be partially replaced by the host-encoded FIS (factor for $i$ nversion stimulation). The bacterial attB consists of a 21-base-pair (bp) core sequence $\left(B O B^{\prime}\right)$ that is composed of 7 central bp, defined as the overlap $O$, which is identical in all four att sites and serves as the site of DNA exchange. $O$ is flanked by two 7-bp inverted repeats $\left(B\right.$ and $\left.B^{\prime}\right)$ that serve as weak binding sites for Int. The phage attP site is composed of a similar core $\left(C O C^{\prime}\right)$ that is flanked by two long arms ( $P$ and $P^{\prime}$ of 142 and $85 \mathrm{bp}$, respectively) carrying tight binding sites for Int and for the accessory proteins (IHF, Xis, and FIS). The accessory proteins, bound to their sites on the arms of attP, regulate and facilitate arm-bound Int monomers access to the core, the site of the reaction. Subsequently, this proteo-DNA complex (the intasome) captures $a t t B$ to execute the recombination. Each of the recombinant sites attL $\left(B O P^{\prime}\right)$ and

\footnotetext{
* Corresponding author. Mailing address: Department of Life Sciences, Ben-Gurion University of the Negev, P.O. Box 653, Be'erSheva 84105, Israel. Phone: 972-8-6461712. Fax: 972-8-6278951. E-mail: burgazla@bgu.ac.il.

${ }^{\nabla}$ Published ahead of print on 8 May 2009.
}

attR $\left(P O B^{\prime}\right)$ that flank the integrated prophage is composed of the recombined core and one of the arms. Since the integration/ excision reactions each use a different pair of att sites and a different composition of the accessory proteins, they are not completely reversible (reviewed in references 1, 25, and 37).

Wild-type Int of $\lambda$-like coliphage HK022 catalyzes its sitespecific integrative and excisive reactions in mammalian cells and in Arabidopsis plants when supplied with the relevant att sites located on a plasmid or on the chromosome $(14,16,21)$. In both reactions, Int does so without the need to supply the accessory proteins IHF and Xis that are required for these reactions in $E$. coli, though supplementation of the accessory factors improves the reaction (22). Int of $\lambda$ can do likewise but only if IHF-independent mutants are active (4). Owing to its versatility in target specificity ( $a t t B$ plus $a t t P$ and $a t t L$ plus $a t t R$ ), the Int system may have an advantage over the common site-specific recombination systems Cre-lox of coliphage P1, FLP-FRT of Saccharomyces cerevisiae, and Int of Streptomyces phage $\phi C 31$ that are already in use for site-specific gene manipulations $(11,15,27,32,34,40)$. Here, we introduced the site-specific recombination system of HK022 into the nitrogenfixing filamentous cyanobacterium Anabaena sp. strain PCC 7120 and demonstrated that Int catalyzes site-specific excision whether its substrate is located on a plasmid or on the chromosome. This system can thus be used as a novel model for simple and efficient genome manipulations in cyanobacteria, in particular, the removal of genes conferring antibiotic resistance, which are unacceptable for applied purposes in transgenic organisms (26).

\section{MATERIALS AND METHODS}

Bacterial strains, plasmids, and primers. The bacterial strains, plasmids, and primers used in this study are listed in Tables 1 and 2. E. coli XL-Blue MRF' 
TABLE 1. Bacterial strains and plasmids used in this study

\begin{tabular}{|c|c|c|}
\hline Strain or plasmid & Genotype or description ${ }^{a}$ & Source or reference \\
\hline \multicolumn{3}{|l|}{ Strains } \\
\hline E. coli XL-Blue $\mathrm{MRF}^{\prime}$ & $\mathrm{F}^{\prime}$ proAB lacl $^{\mathrm{q}} Z \Delta \mathrm{M} 15 \operatorname{Tn} 10\left(\operatorname{Tet}^{\mathrm{r}}\right)$ & Stratagene (1993) \\
\hline E. coli $\mathrm{DH} 10 \mathrm{~B}$ & AM1359 cells for conjugation; carries pRL623 and pRL433 & $\mathrm{X} . \mathrm{Wu}$ \\
\hline $\begin{array}{l}\text { Anabaena sp. strain } \\
\quad \text { PCC } 7120\end{array}$ & Filamentous $\mathrm{N}_{2}$-fixing, internal heterocysts & 38 \\
\hline \multicolumn{3}{|l|}{ Plasmids } \\
\hline pKH3 & int cloned under $P_{t a c}$ on vector pKK233-2 & Lab collection \\
\hline pRL443 & Derivative of conjugal plasmid RP4; $\mathrm{Ap}^{\mathrm{r}} \mathrm{Tet}^{\mathrm{r}} \mathrm{Km}^{\mathrm{s}}$ & 9 \\
\hline pRL528 & Helper plasmid; M.Eco47II, M.AvaI; ColK; $\mathrm{Cm}^{\mathrm{r}}$ & 7 \\
\hline pRL623 & Derivative of pRL528; M.Eco47II, M.AvaI, M.AvaIII & 9 \\
\hline pRL488p & Derivative of pRL488 (8) with $P_{p s b A}$ from pRL435K & 39 \\
\hline pRL488p-int & pRL488p carrying int under $P_{p s b A} ; P_{t a c}$ & This study \\
\hline pLD205 & Carrying the recombination reporter substrate $a t t R-\mathrm{T} 1 / \mathrm{T} 2-a t t L-l a c Z$ & 6 \\
\hline pRL2831a & $\mathrm{Sm}^{\mathrm{r}} \mathrm{Sp}^{\mathrm{r}} ; P_{g l n A} ; \mathrm{RSF} 1010$-based cloning vector & 10 \\
\hline pRL2833b & $\mathrm{Cm}^{\mathrm{r}} \mathrm{Em}^{\mathrm{r}}$; glutamine synthetase promoter $P_{g \ln A}$ & 10 \\
\hline pRSLZ & $\begin{array}{l}\text { Derivative of pRL2831b ( } P_{\text {glnA }} \text { is in the orientation opposite that in pRL2831a), attR-T1/ } \\
\text { T2-attL-lacZ cloned into KpnI-SphI sites of pRL2831b }\end{array}$ & This study \\
\hline pOMs & $\begin{array}{l}\text { Derivative of pRL2833b in which the MluI-MluI fragment was replaced with an intact copy } \\
\text { of all3924 and the SphI-XhoI fragment was replaced with the SphI-XhoI fragment } \\
\text { (carrying } P_{\text {glnA }} \text { attR-T1/T2-attL-lacZ) of pRSLZ }\end{array}$ & This study \\
\hline $\mathrm{pBZ}$ & Derivative of pRSLZ carrying attB-lac $Z$ instead of $a t t R-\mathrm{T} 1 / \mathrm{T} 2-l a c Z$ & This study \\
\hline
\end{tabular}

${ }^{a} \mathrm{Tet}^{\mathrm{r}}$, tetracycline resistance; $\mathrm{Ap}^{\mathrm{r}}$, ampicillin resistance; $\mathrm{Km}^{\mathrm{s}}$, kanamycin sensitivity.

(Stratagene, La Jolla, CA) was used for plasmid constructions. E. coli donor strain DH10B (kindly provided by X. Wu), carrying helper plasmid pRL623 (derivative of pRL528), conjugal plasmid pRL443 (derivative of RP4) (7), and cargo plasmid (either pRL488p-int, pRSLZ, or pOMs), was used for the biparental conjugation (7). Anabaena sp. strain PCC 7120 was employed as the cyanobacterial model system.
The shuttle vector pRL488p (39) was used for cloning int of bacteriophage HK022 into E. coli and Anabaena. The int gene was amplified from plasmid pKH3 by PCR with the primer pair intKpnI and intSacI, and the amplicon was ligated to the KpnI and SacI sites of pRL488p.

Plasmid pRL2831b was constructed by religation of an EcoRI-EcoRI fragment carrying $P_{g \ln A}$ in the opposite orientation into pRL2831a. The recombination

TABLE 2. Primers used in this study

\begin{tabular}{|c|c|c|}
\hline Primer & Sequence $^{a}$ & Purpose \\
\hline int-KpnI & 5'-GGCAGCCATCGGTACCTGTGGATTGGCTG-3' & Amplification of int (complementary to $\mathrm{pKH} 3$ ) \\
\hline int-SacI & 5'-GGGTTATTGTCTCATGAGCTCATACATATTTG-3' & Amplification of int (complementary to $\mathrm{pKH} 3$ ) \\
\hline S-KpnI & 5'-GGAATTGTGAGCGG & Amplification of the recombination substrate (attR-T1/T2-attL-lacZ) \\
\hline S-Sph I & $\begin{array}{l}\text { 5'-ATAATGGAATTCCTTACGCGAAATACGGGCATGC } \\
\text { ATGG-3' }\end{array}$ & Amplification of the recombination substrate (attR-T1/T2-attL-lacZ) \\
\hline 3924uMluI & 5'-GTAAGACATTTTACGCGTGATGAGACGGC-3' & Amplification of all3924 from the genome \\
\hline $3924 \mathrm{~d} M l u \mathrm{I}$ & 5'-GATTTGACGCGTATGGTCAGATAATCGACTTC-3' & Amplification of all 3924 from the genome \\
\hline SeqS1 & 5'-GTTTGA $\overline{C C A A T C A C C C G T C G A A C-3 ' ~}$ & $\begin{array}{l}\text { Sequencing of attR-T1/T2-attL and attB (complementary to the end } \\
\left.\text { of } P_{g \ln A}\right)\end{array}$ \\
\hline SeqS2 & 5'-GGGATGGCTTGTAGATATGACGACAGGAAG-3' & Sequencing of attR-T1/T2-attL \\
\hline SeqS3 & 5'-CTCTAGAAGCTTCGATCTGTGG-3' & Sequencing of $a t t R$-T1/T2-attL \\
\hline SeqS4 & 5'-CCAAGCTTCTAGAGATCTTCCATAC-3' & Sequencing of attR-T1/T2-attL \\
\hline SeqS5 & 5'-GGAAGAGTTTGGTAGAAACG-3' & Sequencing of $a t t R$-T1/T2-attL \\
\hline SeqS6 & 5'-GCTTGGCGTAATCATGGTCATAGCTG-3' & Sequencing of attR-T1/T2-attL \\
\hline SeqP & 5'-GACTGGGAAAACCCTGGCGTTACCC-3' & Sequencing of $a t t B$ \\
\hline$c l$ & 5'-CATTTATACCCTGTGCTATTTGCGTTTTAG-3' & Amplification and sequencing to confirm integration into the genome \\
\hline 3924down2 & 5'-GTGGAAGATAATTATACATAG-3' & Sequencing for integration into the genome \\
\hline$p l$ & 5'-GCCATTGCCTGTAGTACAGACGTTCTTAG-3' & Amplification and sequencing to confirm integration into the genome \\
\hline downZ2 & 5'-GGTACATCTCAAAAAGCTGTC-3' & Sequencing for confirming integration into the genome \\
\hline all(1) & 5'-GTTAAGCTGTGACAAGTGTAATTCTATACCAC-3' & Sequencing of the rescued plasmid \\
\hline $\operatorname{all}(2)$ & 5'-CGGAGAAACCGTCAATGCCAACC-3' & Sequencing of the rescued plasmid \\
\hline $\mathrm{Pg} \ln A$ & 5'-CATTATGGTGAAAGTTGGAACCTC-3' & $\begin{array}{l}\text { Amplification and sequencing before and after excision of pRSLZ or } \\
\text { pBZ }\end{array}$ \\
\hline midlac $Z$ & 5'-GTTCTGCTTCATCTAGAGGATATCCTGCACC-3' & $\begin{array}{l}\text { Amplification and sequencing before and after excision of pRSLZ or } \\
\text { pBZ }\end{array}$ \\
\hline ex1 & 5'-GGAAACAAGATCTAACAATGACTCAACC-3' & $\begin{array}{l}\text { Amplification and sequencing before and after excision from the } \\
\text { chromosome and amplification and sequencing of the } a t t R \text { probe }\end{array}$ \\
\hline$e x 2$ & 5'-GTAACGCCAGGGAGCTCCCAGTCACGACG-3' & $\begin{array}{l}\text { Amplification and sequencing before and after excision from } \\
\text { chromosome }\end{array}$ \\
\hline Ter & 5'-CTTCTAGAGATCTGCAGTCACGCAATAATTAAC-3' & Amplification and sequencing of the $a t t R$ probe \\
\hline lacZ1772 & $5^{\prime}-\mathrm{CG}$ & \\
\hline lacZ2275 & 5'-CACAGCGGATGGTTCGGATAATG-3' & Amplification of the lac $Z$ probe \\
\hline
\end{tabular}

\footnotetext{
${ }^{a}$ The restriction sites are underlined.
} 


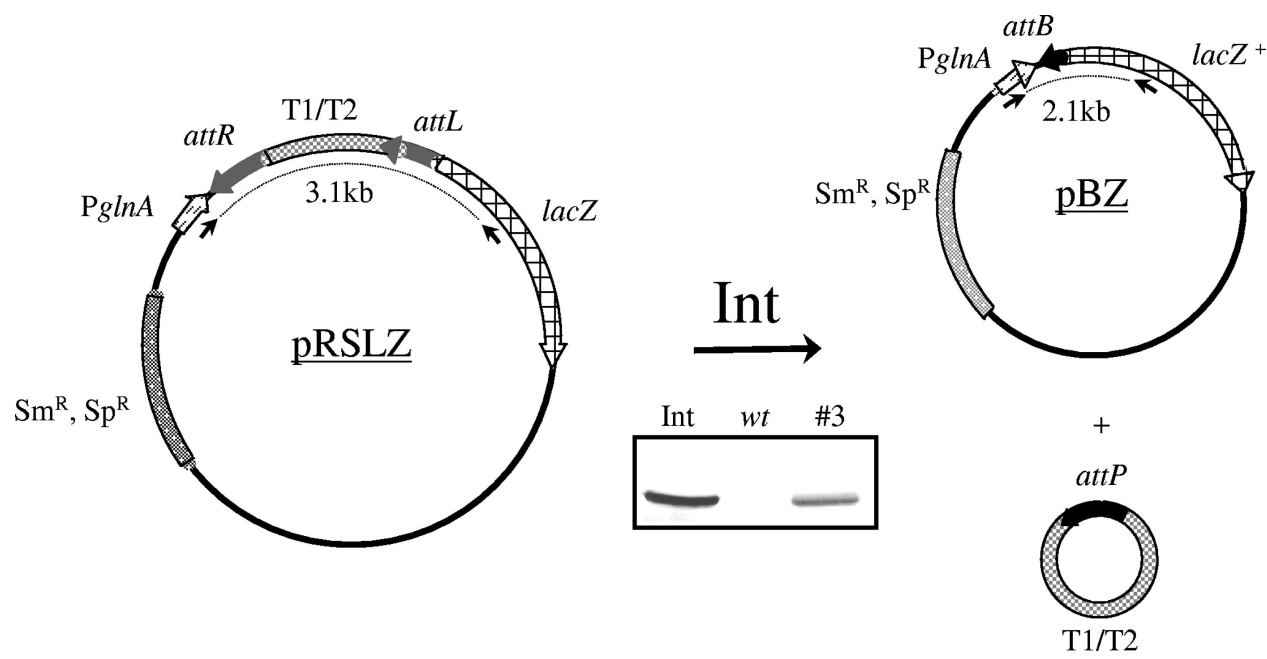

FIG. 1. The recombination reporter plasmid and anticipated products of the Int-catalyzed site-specific excision. (See text for details.) Genes conferring resistance to streptomycin and spectinomycin $\left(\mathrm{Sm}^{\mathrm{r}}\right.$ and $\mathrm{Sp}^{\mathrm{r}}$, respectively) are indicated. (Inset) Western blot analysis of the transgenic Anabaena clone (clone 3) carrying pRL488p-int (lane \#3), Anabaena wild-type cells (lane wt), and E. coli cells expressing int from pKH3 (lane Int). Dotted arcs between arrows indicate amplicons generated with primers Pgln $A$ and midlacZ, which are designated by the small black arrows.

reporter substrate $a t t R$-T1/T2-attL-lac $Z$ was amplified from pLD205 (6) by PCR with primers S-KpnI and S-SphI and cloned between the KpnI and SphI sites of pRL2831b, creating plasmid pRSLZ (Fig. 1). T1/T2 are strong T1 and T2 transcription terminators from the $r m B$ gene (31).

The pOMs plasmid was constructed in two steps as follows: the 1.7-kb MluI fragment (carrying plasmid replication functions in Anabaena) from the pDUI portion of pRL2833b was replaced by the 2.6-kb Anabaena open reading frame all3924, encoding a probable penicillin amidase (see the CyanoBase website [http://bacteria.kazusa.or.jp/cyanobase/index.html]), amplified from the chromosome with the primer pair 3924uMluI and 3924dMluI. The 4,993-bp SphI-XhoI attR-T1/T2-attL-lacZ fragment was then transferred from pRSLZ to the corresponding sites of pRL2833b. pOMs was introduced into Anabaena cells by conjugation. Single recombinants were selected for erythromycin resistance.

Growth conditions. E. coli was grown in Luria-Bertani medium with the following antibiotics and final concentrations: ampicillin, $100 \mu \mathrm{g} \mathrm{ml}^{-1}$; kanamycin, $15 \mu \mathrm{g} \mathrm{ml}^{-1}$; streptomycin, $30 \mu \mathrm{g} \mathrm{ml}^{-1}$; and chloramphenicol, $34 \mu \mathrm{g} \mathrm{ml}^{-1}$.

Anabaena was grown in BG11 medium (38) at $28^{\circ} \mathrm{C}$ under cool white fluorescent light illumination with the following antibiotics and final concentrations: neomycin, $25 \mu \mathrm{g} \mathrm{ml}^{-1}$; spectinomycin, $1.5 \mu \mathrm{g} \mathrm{ml}^{-1}$; and erythromycin, $1 \mu \mathrm{g} \mathrm{ml}^{-1}$.

Conjugation was performed as described by Elhai and Wolk (7).

PCR. Amplification was carried out with Taq DNA polymerase. The DNA in a standard reaction mixture was denatured at $94^{\circ} \mathrm{C}$ for 1 or $5 \mathrm{~min}$ (for plasmid or chromosomal DNA, respectively) and then subjected to 30 cycles of PCR, with 1 cycle consisting of $40 \mathrm{~s}$ at $94^{\circ} \mathrm{C}$ for amplicon denaturation, $40 \mathrm{~s}$ at $53^{\circ} \mathrm{C}$ or $70^{\circ} \mathrm{C}$ for annealing, and $186 \mathrm{~s}$ or $30 \mathrm{~s}$ at $72^{\circ} \mathrm{C}$ for elongation (in plasmid or chromosomal analyses, respectively).

$\boldsymbol{\beta}$-Galactosidase assay. Cells were permeabilized by treatment with chloroform and sodium dodecyl sulfate, and debris was separated by centrifugation after the reaction was terminated as described previously (23). $A_{420}$ was determined in the supernatant, and the specific activity of $\beta$-galactosidase was calculated in terms of $A_{420} \mathrm{~min}^{-1} \mathrm{mg}$ chlorophyll ${ }^{-1}$.

Immunoblot analysis. Proteins (ca. $30 \mu \mathrm{g}$ per lane) were separated by electrophoresis and electrotransferred onto Protran BA 83 cellulose nitrate filters (Schleicher \& Schuell). The blots were exposed to antisera directed against wild-type integrase (20). Protein A-alkaline phosphatase conjugate was used as a primary antibody detector, and bands were visualized using the chromogenic substrate for alkaline phosphatase fast-5-bromo-4-chloro-3-indolylphosphate/nitroblue tetrazolium tablets (Sigma), diluted in $10 \mathrm{ml}$ water.

Southern blot analysis. One microgram of Anabaena genomic DNA was cut with either EcoRI (to confirm substrate integration) or BssHII (to test excisional recombination) and separated on a $0.7 \%$ agarose gel. Alkaline transfer and hybridization were performed according to the GeneScreen manual (DuPont) using ${ }^{32} \mathrm{P}$-labeled probes generated by the random primer method. The following probes were PCR amplified from pLD205: attR fragment with primers ex1 and Ter and part of lacZ with primers lacZ1772 and lacZ2275.
Plasmid rescue. Extracted genomic DNA was cleaved by ClaI, religated, and introduced into E. coli. Transformants were selected on plates containing chloramphenicol.

In vitro site-specific recombination. In vitro site-specific recombination with the reaction mixture containing Int, IHF, Xis, and pRSLZ was performed as previously described (13).

\section{RESULTS}

Int-catalyzed excision from a plasmid. The reporter gene encoding $\beta$-galactosidase (lac $Z$ ) was constructed on a plasmid (pRSLZ, the recombination reporter) such that it is expressed only as a result of an Int-catalyzed site-specific excision reaction (Fig. 1). That is because the Anabaena constitutive promoter for glutamine synthetase $\left(P_{g \ln A}\right)(5,10)$ was separated from the reading frame of lac $Z$ by the strong transcription terminators $\mathrm{T} 1 / \mathrm{T} 2$ flanked by att $R$ and attL in a tandem orientation (6). Int-catalyzed recombination between the two att sites excises the terminator, leading to formation of the 21-bp recombinant $a t t B$ site and brings the $P_{g l n} A$ promoter closer to the reading frame of $l a c Z$, thereby allowing the production of $\beta$-galactosidase (Fig. 1).

The first plasmid (pRL488p-int), carrying int under the tandem constitutive promoters $P_{p s b A}$ and $P_{t a c}$ (39), was introduced into Anabaena by biparental conjugation. Western blot analysis of extracts from an Anabaena exconjugant demonstrated int expression (Fig. 1, inset). The presence of pRL488p-int did not affect the growth rate of the culture (data not shown). Next, the compatible recombination reporter plasmid (pRSLZ) was introduced into the Int expression exconjugant. Int-catalyzed recombination resulted in two products (Fig. 1): attB-containing $\mathrm{pBZ}$ that expresses lacZ $(12.8 \mathrm{~kb})$ and a smaller $(1-\mathrm{kb})$ nonreplicating T1/T2 circular DNA that carries the recombinant attP site.

The recombination activity of Int was monitored in six exconjugants by the expression of lacZ (Fig. 2A) along with cells that carry the substrate alone as a negative control (bar S) and cells transformed with the product alone (bar P). The product 


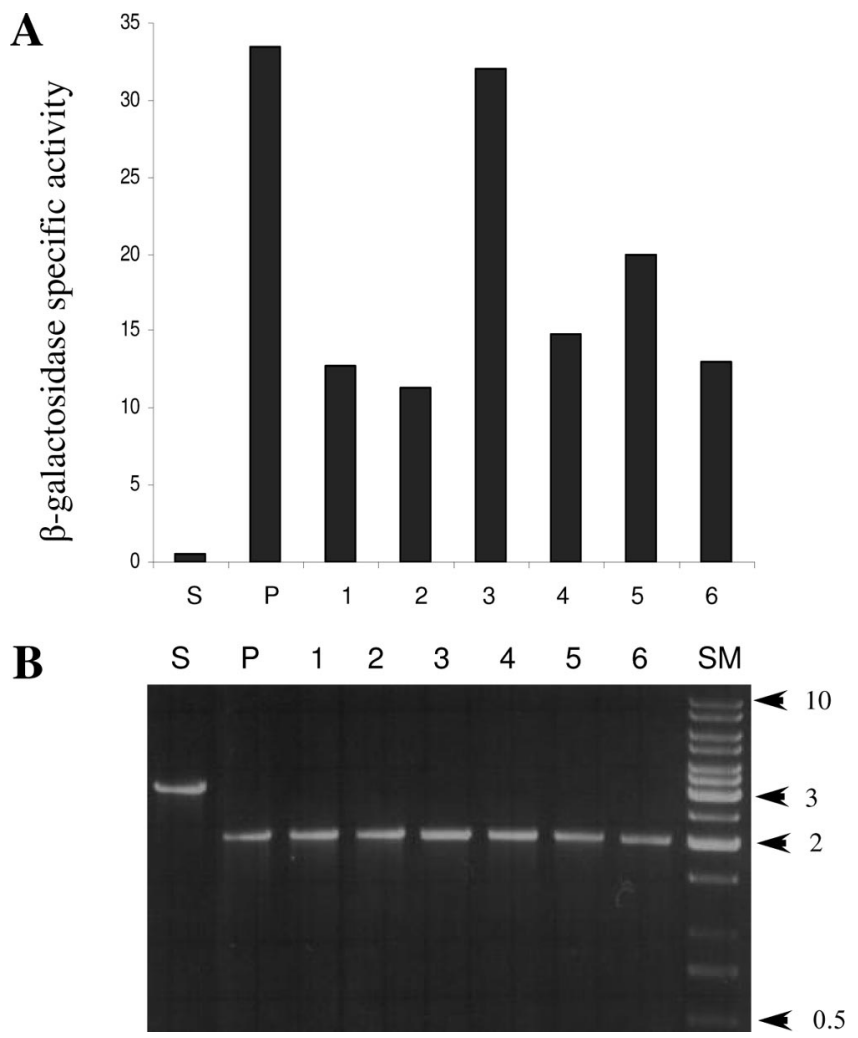

FIG. 2. (A) Specific activities of $\beta$-galactosidase (in $A_{420} \mathrm{~min}^{-1} \cdot \mathrm{mg}$ chlorophyll ${ }^{-1}$ ) in cultures of Anabaena. Clones 1 to 6 (bars 1 to 6 ) carry recombination substrate on pRSLZ and express int from pRL488p-int. Cells with pRSLZ alone (negative control) (bar S) and cells with pBZ product alone (positive control) (bar P) are shown. (B). PCR products from extracted plasmids of the same six transgenic Anabaena clones and controls as shown in panel A. The positions (in kilobases) of size markers (lane SM) are shown to the right of the gel.

(pBZ) was purified from an in vitro excision reaction of the reporter substrate (pRSLZ). Compared to the negative control (bar S), all six Int-treated conjugants expressed LacZ at various amounts, one of them (bar 3 ) as much as the positive control (bar P). The appropriate excision of the T1/T2 fragment was verified by PCR using plasmid extract from each culture (six recombinants and two controls) as template and oligomers $S-K p n I+S-S p h I$ (depicted as arrows in Fig. 1) as primers. Figure $2 \mathrm{~B}$ shows the expected 3.1-kb amplicon from cells with the substrate alone (lane 3) and the expected 2.1-kb amplicon of the recombination product in all other lanes. A sequence analysis (not shown) of all six amplicons obtained from the Inttreated cells confirmed the anticipated presence of the recombinant $a t t B$ site. These results demonstrate that Int functions efficiently in vivo in excisional recombination in Anabaena cells on the episomal level.

Excision from the Anabaena chromosome. To introduce the same recombination substrate $\left(P_{\text {gln }} A^{-a t t R-T 1 / T 2-a t t L-l a c Z}\right)$ into the chromosome by homologous recombination, an Anabaena suicide plasmid, pOMs, that was disabled to replicate in Anabaena, was constructed such that it also carried the 2.6-kb all3924 sequence, a probable penicillin amidase-encoding gene of Anabaena (http://bacteria.kazusa.or.jp/cyanobase/index .html). pOMs was introduced into the Anabaena chromosome via erythromycin selection via a single homologous recombination exchange between its all3924 gene and that of the exconjugant (Fig. 3). The designed integration that results in contiguity of plasmid and genomic DNA was confirmed in recombinants by Southern blotting, PCR analyses, and plasmid rescue. In the blot, genomic DNAs from three erythromycin-recombinant $\left(\mathrm{Em}^{\mathrm{r}}\right)$ recombinants and the wild-type control, each cleaved with EcoRI, were hybridized with an $a t t R$ probe. Figure $3 \mathrm{E}$ shows the expected 12.8 -kb fragment that spans between the inserted plasmid and genome DNA in the left junction and that is absent in the wildtype negative control. Next, the sequence (not shown) of a PCR amplicon obtained with primers $c l$ and $p l$ (Fig. 3C) confirmed the left-hand junction of the integrated plasmid. Finally, genomic DNA from one of the $\mathrm{Em}^{\mathrm{r}}$ recombinants was cut with ClaI and ligated. A chloramphenicol-resistant $\left(\mathrm{Cm}^{\mathrm{r}}\right)$ E. coli transformant of the reaction mixture carried an $11-\mathrm{kb}$ rescue plasmid. Sequence analysis (not shown) of the rescued plasmid using primers all(1) and all(2) revealed the right-hand junction of the integrated plasmid.

The Int-expressing plasmid pRL488p-int was introduced into each of the three recombinant $\mathrm{Em}^{\mathrm{r}}$ clones (Fig. $3 \mathrm{E}$ ) by conjugation, and the Int-catalyzed excision reaction (Fig. $3 \mathrm{C}$ to $3 \mathrm{D})$ was monitored by $\beta$-galactosidase activity in six exconjugants (Fig. 4A, bars 1 to 3, 5, 6, and 8). All six exconjugants displayed $\beta$-galactosidase activity levels about 50 -fold higher than the level of a wild-type Anabaena culture included as a negative control (Fig. 4A, bar 10) and the three transgenic lines not transformed with Int (bars 4, 7, and 9).

Excision of the T1/T2 fragment was evident by PCR of genomic DNA (Fig. 4B) with primers ex1 and ex2 (Fig. 3C and D) in all 10 clones displaying the expected amplicons of 1,244 and 99 bp that represent unrecombined substrate and product, respectively. Sequence analysis (not shown) of the 99-bp amplicons of the Int-treated cultures confirmed the recombinant $a t t B$ sequence. Finally, the expected 3- and 2-kb fragments of BssHII-cleaved genomic DNA prior to and following excision (Fig. 3C and D) were confirmed by Southern blot analysis (Fig. $3 \mathrm{~F}$, lanes 3 and 4, respectively) using a 508-bp lac $Z$ probe. The additional, intense bands in lane 4 of Fig. $3 \mathrm{~F}$ probably indicate incomplete digestion of the genomic DNA. These results show that Int efficiently catalyzed the excision of the substrate when located on a chromosome.

\section{DISCUSSION}

Expression of the coliphage HK022 int and the site-specific excisive recombination activity of its product in the cyanobacterium Anabaena sp. strain PCC 7120 are reported here for the first time. A similar study with Cre with two separately inserted chromosomal lox sites was reported earlier (40). Int was active with no need to import the accessory proteins IHF and Xis that are required for its activity in its natural E. coli host. The Int-catalyzed site-specific recombination, monitored by the expression of $\beta$-galactosidase production, was evident whether the recombination substrate was located on a plasmid (Fig. 1 and 2) or on the chromosome (Fig. 3 and 4). For the extrachromosomal reaction, int and the substrate were consecutively introduced into Anabaena cells on separate plasmids. In the case of the chromosomal reaction, the Int-encoding plasmid was introduced into transgenic strains carrying the recombina- 

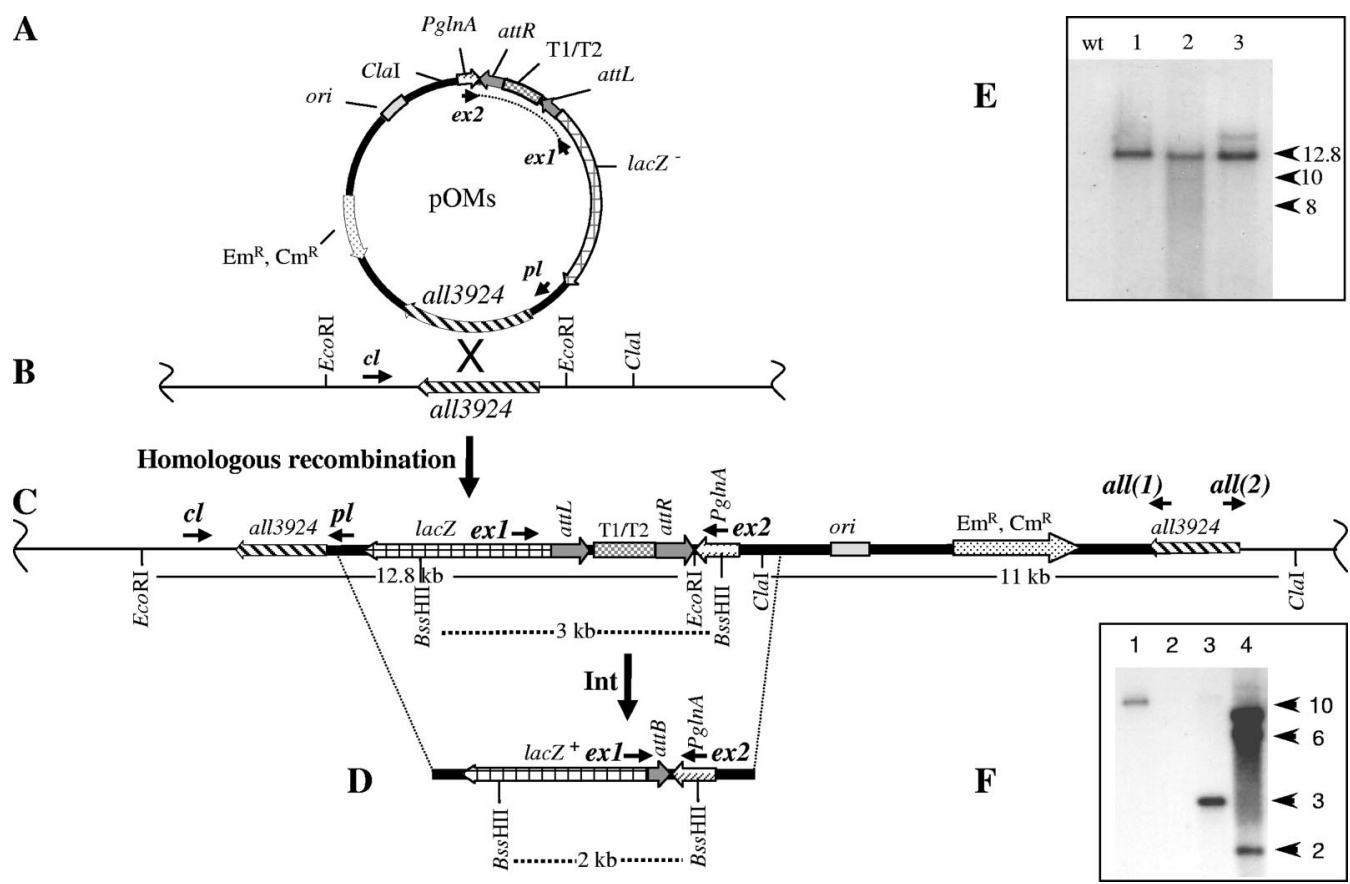

FIG. 3. (A to D) Schematic representation of the pOMs plasmid (A), the Anabaena chromosome (B), their homologous recombination product (C), and the following site-specific excision product (D). ori, replication origin in E. coli. Genes conferring resistance to erythromycin and chloramphenicol $\left(\mathrm{Em}^{\mathrm{r}}\right.$ and $\mathrm{Cm}^{\mathrm{r}}$, respectively) are indicated. The small black arrows indicate primers used in PCRs and sequencing. (E) Southern blot of three $\mathrm{Em}^{\mathrm{r}}$ recombinant Anabaena clones (lanes 1 to 3) and of the wild-type (wt) Anabaena as a negative control. Hybridization was against ${ }^{32}$ P-labeled attR probe. (F) Southern blot of wild-type Anabaena DNA (lane 2), a clone that carries the chromosomal recombination substrate that was not treated with Int (lane 3), and a similar Int-treated clone (lane 4). Lane 1 contains a linearized plasmid carrying the recombination substrate as a positive control. A lacZ fragment was used as the probe. The positions of molecular size markers (in kilobases) are indicated by black arrowheads to the right of the gels in panels $\mathrm{E}$ and F. Further details are described in the text.

tion substrate. The site-specific excision was confirmed in both cases by the formation of the recombined $a t t B$ site. All six recombinant clones obtained by either the extrachromosomal or chromosomal reaction showed only the product and no substrate cassette (Fig. 2B and 4B), indicating a high frequency of recombination. This is in contrast to the relatively lower activity of Int observed in mammalian and plant cells $(14,16)$. In the case of the extrachromosomal reactions, this high frequency could be explained by the fact that the exconjugants were selected in succession, for the Int-encoding plasmid (pRL488p-int) first, followed by the low-copy-number plasmid encoding the recombination substrate (pRSLZ) (Fig. 1). Since int had been constitutively expressed during many generations of cell growth before Int activity was tested, the presence of Int in cells with the newly introduced substrate may be the reason for the high frequency of recombination. Also, in the chromosomal excision, where the Int-encoding plasmid was introduced to transgenic cells containing the substrate on the chromosome, many generations of selection elapsed before the reaction was examined. However, it should also be noticed that in the chromosomal reaction, the size of the excision product of 99 bp was about 12.5 -fold smaller than of the substrate $(1,244$ bp; Fig. 4B), such that the former may have preferentially replicated. The same can also partially hold for the extrachromosomal PCR analysis (Fig. 2B). Likewise, the Southern blot analysis (Fig. 3F) demonstrates that the clone expressing int has exposed only the $2-\mathrm{kb}$ product but not the $3-\mathrm{kb}$ substrate, supporting the assumption that the excisional recombination is effective in Anabaena.

The variation in levels of $\beta$-galactosidase activity was much higher in the six clones in which the recombination substrate was on a plasmid (Fig. 2A) than in the six clones where it was chromosomally located (Fig. 4A). This variation may have resulted from a varying number of product ( $\mathrm{pBZ}$ ) copies per cell, whereas the frequency of the excised chromosome is stable. It is noteworthy that spectinomycin selection was partial: nonrecombinant cultures of Anabaena survived in the presence of the low drug concentrations used for the selection, albeit at a lower growth rate (data not shown).

Integration and excision catalyzed by Int of coliphage HK022 without the supplementary accessory proteins that are required in the natural host have already been demonstrated in mammalian cells (21) and Arabidopsis plants (14), where they may be substituted by endogenous, nonspecific DNA-bending proteins HMG1/2 (33). It is thus likely that proteins substituting for IHF and Xis are present in the cyanobacterium as well. However, sequences homologous to $E$. coli's IHF-encoding subunit genes (ihf $A$ and $i h f B$ ) and to fis do not exist in the Anabaena sp. strain PCC 7120 genome, leaving the apparent substitutes enigmatic.

The small basic E. coli protein HU (29) encoded by hupA or hupB serves as a chaperone to bend and loop DNA $(2,36)$. IHF binds to specific sequences by forming structural properties of the DNA, whereas HU binds to structural distortions in 
A

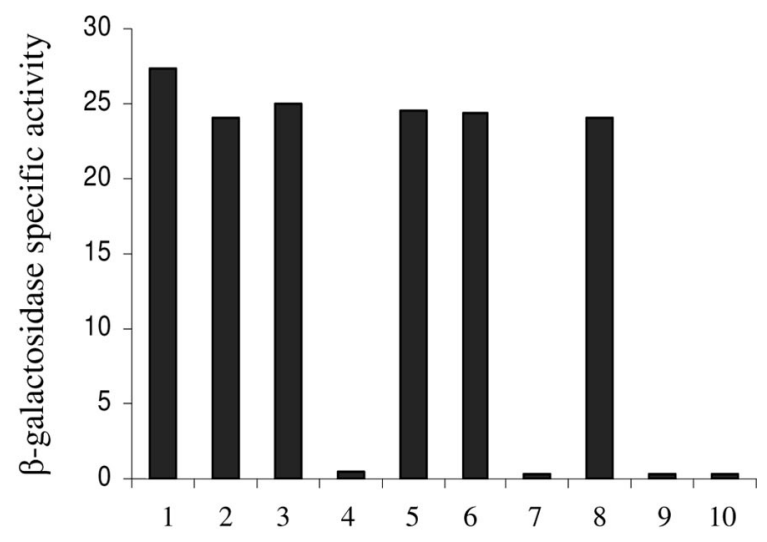

B

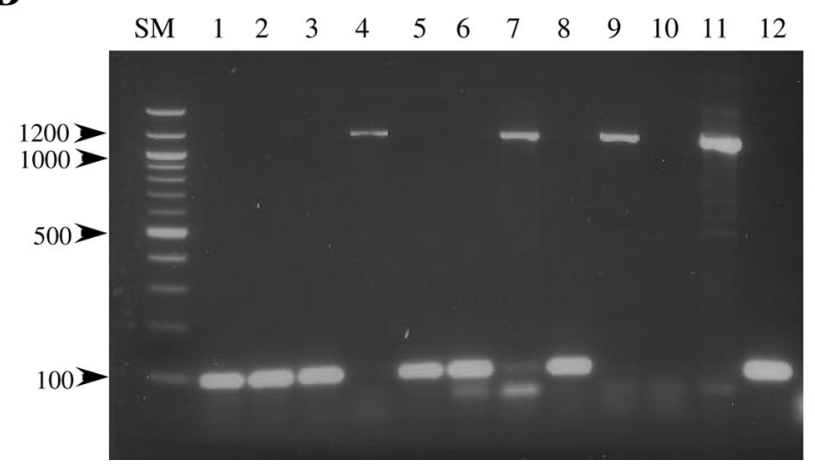

FIG. 4. (A) Specific activities of $\beta$-galactosidase (in $A_{420} \mathrm{~min}^{-1} \cdot \mathrm{mg}$ chlorophyll $^{-1}$ ) in cultures of six transgenic Anabaena clones carrying the recombination substrate on the chromosome and expressing int from pRL488p-int. Exconjugants stemming from introduction of pRL488p-int into clone 1 of Fig. 3E (bars 1 to 3), exconjugants from clone 2 of Fig. 3E (bars 5 and 6), an exconjugant from clone 3 of Fig. $3 \mathrm{E}$ (bar 8), and clones 1 to 3 of Fig. 3E not treated with Int (negative controls) (bars 4, 7, and 9), and the wild-type Anabaena clone (bar 10) are shown. (B) PCR analysis of genomic DNA extracted from Anabaena. Lanes 1 to 10 show the amplicons from the respective transgenic clones as in panel $\mathrm{A}$; lanes 11 and 12 contain pure pOMs (substrate [Fig. 3]) and pBZ (product [Fig. 1]); lane SM, 100-bp ladder (size markers).The primers were ex1 and ex2. The positions (in base pairs) of molecular size markers are shown to the left of the gel by the black arrowheads.

DNA irrespective of sequence (30). Nevertheless, HU can replace IHF in $\lambda$ site-specific excisive recombination (12); both have closely related structures and similar mechanisms of DNA bending $(18,35)$. The Anabaena HU-like protein (17), encoded by han $A$ (24), is essential for sensitivity to cyanophage A-4(L) and heterocyst differentiation (19). It is synthesized in the vegetative cells, but not in the heterocysts, and is degraded during differentiation (24). Integrating this information leads to the possibility that the Anabaena HanA might substitute for the functions of IHF and Xis. Alternatively, the site-specific recombinase XisF of Anabaena sp. strain PCC 7120 (3), which is involved in regulating $f d x N$ during heterocyst differentiation, requires two additional accessory proteins ( $\mathrm{XisH}$ and $\mathrm{XisI}$ ) whose functions are yet to be disclosed (28).

The independence of HK022 Int activity on any imported accessory protein in Anabaena and its extraordinary high efficiency can be useful as a novel effective tool for DNA manipulations in Anabaena and other cyanobacteria, for example, to remove genes for antibiotic resistance from transgenic strains designed for release in nature (e.g., Agmenellum quadruplicatum PR-6, Synechocystis sp. strain PCC 6803, Synechococcus sp. strain PCC 7942, and Anabaena sp. strain PCC 7120) that express one or a combination of genes for mosquito larvicidal proteins from Bacillus thuringiensis subsp. israelensis (26).

\section{ACKNOWLEDGMENTS}

We thank C. Peter Wolk for providing pRL2831a and pRL2833b and for helpful advice on integrating plasmid DNA into the Anabaena sp. strain PCC 7120 chromosome. In addition, we thank Vadim Khasdan and Monice Einav for helpful advice.

This investigation was supported by grants from the United StatesIsrael Binational Science Foundation, Jerusalem, Israel (grant 2007037 to A.Z. and grant 2003394 to E.Y. and M.K.) and by an Eshkol Fellowship (grant 82606101) from the Israel Ministry of Science, Jerusalem (to O.M.).

\section{REFERENCES}

1. Azaro, M. A., and A. Landy. 2002. Integrase and the $\lambda$ int family, p. 118-148. In N. L. Craig, R. Craigie, M. Gellert, and A. Lambowitz (ed.), Mobile DNA II. American Society for Microbiology, Washington, DC.

2. Bianchi, M. E. 1994. Prokaryotic HU and eukaryotic HMG1: a kinked relationship. Mol. Microbiol. 14:1-5.

3. Carrasco, C. D., K. S. Ramaswamy, T. S. Ramasubramanian, and J. W. Golden. 1994. Anabaena xis $F$ gene encodes a developmentally regulated site-specific recombinase. Genes Dev. 8:74-83.

4. Christ, N., and P. Dröge. 2002. Genetic manipulations of mouse embryonic stem cells by mutant $\lambda$ integrase. Genesis 32:203-208.

5. Cohen-Kupiec, R., M. Gurevitz, and A. Zilberstein. 1993. Expression of $\operatorname{gln} A$ in the cyanobacterium Synechococcus sp. strain PCC 7942 is initiated from a single nif-like promoter under various nitrogen conditions. J. Bacteriol. 175: 7727-7731.

6. Dorgai, L., E. Yagil, and R. Weisberg. 1995. Identifying determinants of recombination specificity: construction and characterization of mutant bacteriophage integrases. J. Mol. Biol. 252:178-188.

7. Elhai, J., and C. P. Wolk. 1988. Conjugal transfer of DNA to cyanobacteria Methods Enzymol. 167:747-754.

8. Elhai, J., and C. P. Wolk. 1990. Developmental regulation and spatial pattern of expression of the structural genes for nitrogenase in the cyanobacterium Anabaena. EMBO J. 9:3379-3388.

9. Elhai, J., A. Vepritskiy, A. M. Muro-Pastor, E. Flores, and C. P. Wolk. 1997. Reduction of conjugal transfer efficiency by three restriction activities of Anabaena sp. strain PCC 7120. J. Bacteriol. 179:1998-2005.

10. Fan, Q., G. Huang, S. Lechno-Yossef, C. P. Wolk, T. Kaneko, and S. Tabata. 2005. Clustered genes required for synthesis and deposition of envelope glycolipids in Anabaena sp. strain PCC 7120. Mol. Microbiol. 58:227-243.

11. Gilbertson, L. 2003. Cre-lox recombination: Cre-ative tools for plant biotechnology. Trends Biotechnol. 21:550-555.

12. Goodman, S. D., S. C. Nicholson, and N. A. Nash. 1992. Deformation of DNA during site-specific recombination of bacteriophage A: replacement of IHF protein by HU protein or sequence-directed bends. Proc. Natl. Acad. Sci. USA 89:11910-11914.

13. Gottfried, P., M. Kolot, and E. Yagil. 2001. The effect of mutations in the Xis-binding sites on site-specific recombination in coliphage HK022. Mol. Gen. Genomics 266:584-590.

14. Gottfried, P., O. Lotan, M. Kolot, L. Maslenin, R. Bendov, R. Gorovits, V. Yesodi, E. Yagil, and A. Rosner. 2005. Site-specific recombination in Arabidopsis plants promoted by integrase protein of coliphage HK022. Plant Mol. Biol. 57:435-444.

15. Groth, A. C., and M. P. Calos. 2004. Phage integrases: biology and applications. J. Mol. Biol. 335:667-678.

16. Harel-Levi, G., J. Goltsman, C. N. Tuby, E. Yagil, and M. Kolot. 2008 Human genomic site-specific recombination catalyzed by coliphage HK022 integrase. J. Biotechnol. 134:46-54.

17. Haselkorn, R., and J. Rouviere-Yaniv. 1976. Cyanobacterial DNA-binding protein related to Escherichia coli HU. Proc. Natl. Acad. Sci. USA 73:19171920.

18. Johnson, R. C., L. A. Johnson, J. W. Schmidt, and J. F. Gardner. 2005. Major nucleoid proteins in the structure and function of the Escherichia coli chromosome, p. 65-132. In N. P. Higgins (ed.), The bacterial chromosome. American Society for Microbiology, Washington, DC.

19. Khudyakov, I., and C. P. Wolk. 1996. Evidence that the hanA gene coding for HU protein is essential for heterocyst differentiation in, and cyanophage A-4(L) sensitivity of, Anabaena sp. strain PCC 7120. J. Bacteriol. 178:35723577 .

20. Kolot, M., N. Silberstein, and E. Yagil. 1999. Site-specific recombination in mammalian cells expressing the Int recombinase of bacteriophage HK022. Mol. Biol. Rep. 26:207-213. 
21. Kolot, M., A. Meroz, and E. Yagil. 2003. Site-specific recombination in human cells catalyzed by the wild-type integrase protein of coliphage HK022. Biotechnol. Bioeng. 84:6-60.

22. Malchin, N., J. Goltsman, L. Dabool, R. Gorovits, Q. Bao, P. Dröge, E. Yagil, and M. Kolot. 2009. Optimization of coliphage HK022 integrase activity in human cells. Gene 437:9-13.

23. Miller, J. H. 1972. Experiments in molecular genetics. Cold Spring Harbor Laboratory, Cold Spring Harbor, NY.

24. Nagaraja, R., and R. Haselkorn. 1994. Protein HU from the cyanobacterium Anabaena. Biochimie 76:1082-1089.

25. Nash, H. A. 1996. Site-specific recombination: integration, excision, resolution, and inversion of defined DNA segments, p. 2363-2376. In F. C. Neidhardt, R. Curtiss III, J. L. Ingraham, E. C. C. Lin, K. B. Low, B. Magasanik, W. S. Reznikoff, M. Riley, M. Schaechter, and H. E. Umbarger (ed.), Escherichia coli and Salmonella: cellular and molecular biology. American Society for Microbiology, Washington, DC.

26. Otieno-Ayayo, Z. N., A. Zaritsky, M. C. Wirth, R. Manasherob, V. Khasdan, R. Cahan, and E. Ben-Dov. 2008. Variations in the mosquito larvicidal activities of toxins from Bacillus thuringiensis ssp. israelensis. Environ. Microbiol. 10:2191-2199.

27. Puchta, H. 2003. Towards the ideal GMP: homologous recombination and marker gene excision. J. Plant Physiol. 160:743-754.

28. Ramaswamy, K. S., C. D. Carrasco, T. Fatmat, and J. W. Golden. 1997. Cell-type specificity of the Anabaena $f d x N$-element rearrangement requires $x i s H$ and xisI. Mol. Microbiol. 23:1241-1250.

29. Rouviere-Yaniv, J., and F. Gros. 1975. Characterization of a novel, low molecular weight DNA-binding protein from Escherichia coli. Proc. Natl. Acad. Sci. USA 72:3428-3432.

30. Rowland, S. J., M. R. Boocock, and W. M. Stark. 2006. DNA bending in the Sin recombination synapse: functional replacement of HU by IHF. Mol. Microbiol. 59:1730-1743.
31. Sarmientos, P., J. E. Sylvester, S. Contente, and M. Cashel. 1983. Differential stringent control of the tandem $E$. coli ribosomal RNA promoters from the rrnA operon expressed in vivo in multicopy plasmids. Cell 32:1337-1346.

32. Scutt, C. P., E. Zubko, and P. Meyer. 2002. Techniques for the removal of marker genes from transgenic plants. Biochimie 84:1119-1126.

33. Segall, A. M., S. D. Goodman, and H. A. Nash. 1994. Architectural elements in nucleoprotein complexes: interchangeability of specific and non-specific DNA binding proteins. EMBO J. 13:4536-4548.

34. Sorrell, D. A., and A. F. Kolb. 2005. Targeted modification of mammalian genomes. Biotechnol. Adv. 23:431-469.

35. Swinger, K. K., and P. A. Rice. 2004. IHF and HU: flexible architects of bent DNA. Curr. Opin. Struct. Biol. 14:28-35.

36. Travers, A. M., S. S. Ner, and M. E. A. Churchill. 1994. DNA chaperones: a solution to a persistence problem? Cell 77(2):167-169.

37. Weisberg, R. A., M. E. Gottesman, R. W. Hendrix, and J. W. Little. 1999. Family values in the age of genomics: comparative analyses of temperate bacteriophage HK022. Annu. Rev. Genet. 33:565-602.

38. Wolk, C. P., A. Vonshak, P. Kehoe, and J. Elhai. 1984. Construction of shuttle vectors capable of conjugative transfer from Escherichia coli to nitrogen-fixing filamentous cyanobacteria. Proc. Natl. Acad. Sci. USA 81: 1561-1565.

39. Wu, X., S. J. Vennison, L. Huirong, E. Ben-Dov, A. Zaritsky, and S. Boussiba. 1997. Mosquito larvicidal activity of transgenic Anabaena strain PCC 7120 expressing combinations of genes from Bacillus thuringiensis subsp. israelensis. Appl. Environ. Microbiol. 63:4971-4975.

40. Zhang, Y., H. Pu, Q. Wang, C. Cheng, W. Zhao, Y. Zhang, and J. Zhao. 2007. PII is important in regulation of nitrogen metabolism but not required for heterocyst formation in the cyanobacterium Anabaena sp. PCC 7120. Biol Chem. 282:33641-33648 